\title{
Strategies to Reduce Alcohol-Exposed Pregnancies
}

\author{
R. Louise Floyd · Shahul Ebrahim • James Tsai • \\ Mary O'Connor · Robert Sokol
}

Published online: 22 July 2006

(C) Springer Science+Business Media, Inc. 2006

Keywords Alcohol $\cdot$ Pregnancy $\cdot$ Preconception care

\section{Introduction}

Prenatal alcohol exposure remains a leading preventable cause of birth defects and developmental disabilities in the United States, with the prevalence of alcohol consumption by women in childbearing age remaining high and unchanged over time (Fig. 1). This paper provides a summary of current knowledge and information on recognition and prevention of an alcohol-exposed pregnancy, including overall alcohol exposure burden among women of child-bearing age, diag-

Contributions: RLF conceived the idea, RLF and SE developed the framework for the paper, and JT conducted the statistical analyses and developed the figure. RLF, SE, MO and RS contributed to the writing and reviewed the content. All authors reviewed, commented, and contributed to the final version of the manuscript.

Conflicts of interest: None declared.

R. L. Floyd · S. Ebrahim · J. Tsai

Centers for Disease Control \& Prevention,

Atlanta, Georgia

\section{O'Connor}

Department of Psychiatry and Biobehavioral Sciences, University of California at Los Angeles, David Geffen School of Medicine, Los Angeles, California

R. Sokol

Department of Obstetrics and Gynecology, Wayne State University, School of Medicine,

Detroit, Michigan

S. Ebrahim $(\bowtie)$

Centers for Disease Control \& Prevention (E86),

Atlanta, Georgia, 30333

e-mail: sebrahim@cdc.gov nostic criteria for fetal alcohol syndrome (FAS), and recommendations for screening, assessment and interventions to reduce alcohol exposed pregnancies.

\section{Burden of alcohol exposure in pregnancy}

Most women reduce their alcohol use substantially when they realize they are pregnant, but significant numbers continue to drink at levels that can be hazardous to the fetus $[1,2]$. Of particular concern are the group of sexually active women who are not planning to become pregnant (about half of all pregnancies in the US), but do so and continue alcohol use during the early stages of embryonic and fetal development [1]. For many women, pregnancy recognition does not occur until the 6th week of gestation [3]. According to national survey data, in 2002 about $8 \%$ of women aged 18 to 44 years were sexually active, fertile, not using any form of birth control, and at risk of becoming pregnant [1]. This group of women also report high rates of binge drinking, with 1 in 5 in the age category of 18 to 24 years reporting multiple episodes of binge drinking, and are therefore at risk for an alcohol-exposed pregnancy.

\section{Adverse fetal outcomes}

Prenatal alcohol exposure can result in a wide range of adverse outcomes including fetal alcohol syndrome (FAS), although not all infants exposed to alcohol in utero develop the same severity of effects as do others. The term fetal alcohol spectrum disorder (FASD) has been introduced to describe the range of physical, mental and behavioral effects that can occur in an individual exposed to alcohol in utero. Because diagnostic criteria are not available for all conditions along 


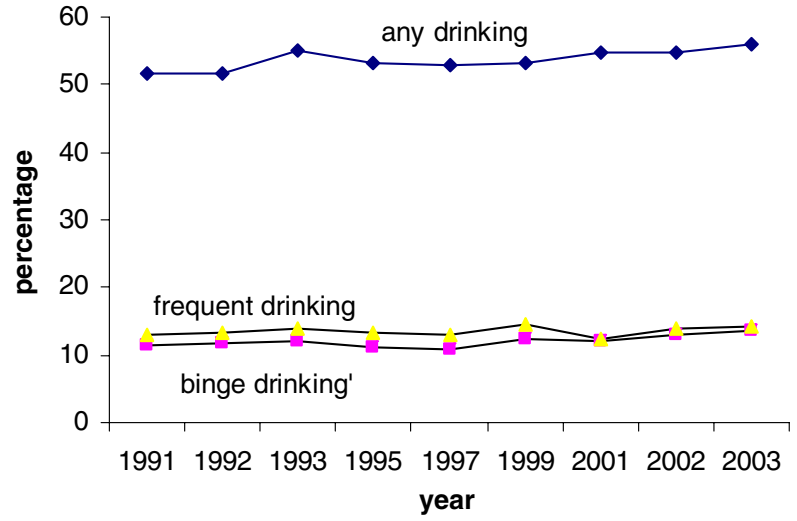

Fig. 1 Weighted percentage of alcohol consumption for non-pregnant women aged 18 to 44 years during the previous 30 days, BRFSS, United States. Data were not collected in 1994, 1996, 1998, and 2000. Frequent refers to $=7$ drinks/week or binge. Binge drinking refers to $=5$ drinks on one occasion

the spectrum, there are no prevalence rates available for the full spectrum. Prevalence rates of FAS range from 0.3 to 2.0 cases per 1,000 live births [4] depending on the methodology used, and the sub-populations assessed. Reported rates are higher among infants born to women who are American Indian/Alaska Native or African American, unmarried, smokers, have low incomes, and have a history of previous drug use or mental health conditions [5, 6]. In an effort to promote the more complete recognition of FAS, guidelines for identifying and referring persons with FAS have been collaboratively developed and disseminated recently [4] (www.cdc. gov/ncbddd/fas/documents/fas_guidelines_accessible.pdf).

\section{Approaches to reducing alcohol-exposed pregnancies}

A 1996 report from the Institute of Medicine addressing prevention of FAS recommended implementation of preventative actions at multiple levels including individual, group, and universal levels [7]. To date, most of the effective prevention strategies identified have focused on the individual level. In 2004 the U.S. Preventive Services Task Force (USPSTF) released a report recommending screening and behavioral interventions to reduce alcohol misuse in adults in primary care settings [8], www.preventiveservices.ahrq.gov.

The report concluded that effective interventions include brief counseling comprised of feedback regarding screening and assessment information, advice, goal-settings and follow-up assistance. The report further cited complementary practices of motivational interviewing, assessing readiness to change, and use of the 5 A's behavioral counseling framework of assess, advice, agree, assist and arrange. Validated screening instruments are available for screening pregnant and non-pregnant women of reproductive age including the T-ACE, TWEAK, and AUDIT [9]. More information on these instruments is available at the following website: www.nih.gov/publications/Assessing/Alcohol/Index.htm.

Primary healthcare providers can play a pivotal role in identifying women of reproductive age (during pregnancy and before pregnancy occurs) who are at high risk for an alcohol-exposed pregnancy and providing them with advice, counseling, and referral as appropriate. This strategy has widespread support among professional organizations including the American College of Obstetricians and Gynecologist (ACOG), and the American Academy of Pediatrics (AAP), as well the U.S. Office of the Surgeon General, and the U.S. Department of Health and Human Services.

A useful example of an intervention targeting groups of women using many of the components described in the recommendation of the USPSTF is found in a CDC sponsored study, Project CHOICES, a feasibility pilot study that targeted non-pregnant women at high risk for an alcoholexposed pregnancy [10]. Project CHOICES was conducted in diverse sub-populations of women determined to have higher proportions of individuals at risk for an alcoholexposed pregnancy. Compared to the overall estimated $2 \%$ of childbearing women at risk for an alcohol-exposed pregnancy, the Project CHOICES intervention settings (e.g. a jail, alcohol and drug treatment facilities, and primary care clinics) had an overall rate of $12.5 \%$ of women at risk for an alcohol-exposed pregnancy. A unique aspect of the intervention is that it focused not only on reducing risk drinking, but also addressed pregnancy postponement as a route for avoiding an alcohol-exposed pregnancy. The intervention consisted of 4 brief motivational interventions sessions and 1 consultation visit to a family planning provider. All participants were at risk for an alcohol-exposed pregnancy in that they were sexually active, fertile, risky drinkers and not taking effective measures to avoid pregnancy. At the 6 month follow-up assessment, $68.5 \%$ were at reduced risk because they had either changed their risk drinking, instituted effective contraception, or both. Subsequently, the Project CHOICES Research Group completed a randomized controlled trail to test the efficacy of the intervention with a report of the study findings currently underway.

Universal level interventions have not received recent attention with the exceptions of the 2005 release of U.S. Surgeon General's Advisory on Alcohol Use and Pregnancy, http://www.hhs.gov/surgeongeneral/pressrelease/sg0222205. $\mathrm{html}$. This advisory drew attention to the continuing problem of FASD and the continuing high rates of alcohol use, including binge drinking, among childbearing aged women in the U. S. The impact of the advisory has provided support for those seeking to inform and educate the public healthcare system overall to this important public health concern. 


\section{Summary}

In addition to the adverse effects of alcohol on the fetus, alcohol leads also to many other adverse effects on the health of women including reproductive health conditions that are not addressed in this brief. Though the approaches mentioned here are primarily aimed at reduction of prenatal alcohol-related pregnancies, any reduction in hazardous alcohol consumption among women will add to improvements in the general health of women. Multiple federal and non-fedral health agencies and organizations have recommended that pregnant women and those planning a pregnancy abstain from alcohol use. Although assessment and interventions are valuable tools to recognize and address alcohol use and secondary pregnancy outcomes, they are underutilized in primary care settings. To reduce the burden of alcohol exposed pregnancies and alcohol's impact on the health of families, it is critical for physicians and health care providers to consistently screen childbearing-aged women for alcohol use with validated screening tools which can be embedded in the patient screening protocol. Tools are now available for pediatricians and child health care providers to enhance early recognition of FASD and reduce secondary conditions that often accompany physical maturation [4]. Brief clinician-delivered behavioral interventions to women and their partners, counseling regarding effective contraceptive options when not planning a pregnancy, and improving access to such services for those who are unable reduce their alcohol intake can help more women reduce their risk for an alcohol exposed pregnancy [11]. Referral to needed social services should complement health services in order to achieve maximum benefits of primary care-based attempts to reduce alcohol-exposed pregnancies. Given the levels of alcohol use among childbearing aged women, primary care- based individual level assessment and intervention continues to remain a critical prevention strategy for reducing alcoholexposed pregnancies.

Acknowledgement We thank the anonymous reviewers for their comments.

\section{References}

1. CDC. Alcohol use among women of childbearing age - United States, 1991-1999. MMWR. 2002;52:273-6.

2. Ebrahim SH, Gfroerer. Pregnancy-related substance use in the United States during 1996-1998. Obstet Gynecol 2003;101:3749.

3. Floyd RL, Decoufle P, Hungerford DW. Alcohol use prior to pregnancy recognition. Am J Prev Med 1999;17:101-7.

4. CDC. Guidelines for identifying a person with fetal alcohol syndrome. $M M W R$. 2005;54(No. RR-11).

5. CDC. Fetal alcohol syndrome -Alaska, Arizona, Colorado, and New York, 1995-1997. MMWR. 2002;51:433-35.

6. May PA, Gossage JP. Estimating the prevalence of fetal alcohol syndrome: a summary. Alcohol, Research \& Health. 2001;25;1597.

7. Stratton K, Howe C, Battaglia F, editors. Fetal Alcohol Syndrome: Diagnosis, Epidemiology, Prevention, and Treatment. 1996. Institute of Medicine. National Academy Press. Washington, DC.

8. U.S. Preventive Services Task Force. Screening and behavioral counseling intervention in primary care to reduce alcohol misuse: recommendations statement. Ann Intern Med. 2004:140:554-6.

9. Floyd RL, O'Connor MJ, Sokol RJ, Bertrand J, Cordero JF. Recognition and prevention of fetal alcohol syndrome. Obstet Gynecol. 2005;106(5):1059-64.

10. The Project CHOICES Intervention Research Group. Reducing the risk of alcohol-exposed pregnancies: a study of a motivational intervention in community settings. Pediatrics. 2003;111(5):11315.

11. Chang G, McNamara TK, Orav EJ, Koby D, Lavigne A, Ludman G, Vincitorio NA, Wilkins-Haug L. Brief interventions for prenatal alcohol use: a randomization trial. Obstet Gynecol. 2005;105: 991-8. 